Acta Crystallographica Section E

Structure Reports

Online

ISSN 1600-5368

\section{Amodiaquinium dichloride dihydrate from laboratory powder diffraction data}

Antonio Llinàs, ${ }^{a}$ László Fábián, ${ }^{b}$ Jonathan C. Burley, Jacco van de Streek ${ }^{d}$ and Jonathan M. Goodman ${ }^{\mathrm{a} *}$

${ }^{a}$ Unilever Centre for Molecular Informatics, Pfizer Institute for Pharmaceutical Materials Science, Department of Chemistry, University of Cambridge, Lensfield Road, Cambridge CB2 1EW, England, ${ }^{\mathbf{b}}$ Pfizer Institute for Pharmaceutical Materials Science, Cambridge Crystallographic Data Centre, 12 Union Road, Cambridge CB2 1EZ, England, 'University Chemical Laboratory, Lensfield Road, Cambridge CB2 1EW, England, and ${ }^{\mathbf{d}}$ Cambridge Crystallographic Data Centre, 12 Union Road, Cambridge CB2 1EZ, England

Correspondence e-mail: fabian@ccdc.cam.ac.uk

Key indicators

Powder X-ray study

$T=298 \mathrm{~K}$

Mean $\sigma(\mathrm{C}-\mathrm{C})=0.008 \AA$

$R$ factor $=0.037$

$w R$ factor $=0.047$

Data-to-parameter ratio $=0.0$

For details of how these key indicators were automatically derived from the article, see http://journals.iucr.org/e.
The title compound (systematic name: $\{5-[(7-$ chloroquinolinium-4-yl)amino]-2-hydroxybenzyl dimethylammonium dichloride dihydrate), $\mathrm{C}_{20} \mathrm{H}_{24} \mathrm{ClN}_{3} \mathrm{O}^{2+} \cdot 2 \mathrm{Cl}^{-}$$.2 \mathrm{H}_{2} \mathrm{O}$, has one amodiaquinium dication, two $\mathrm{Cl}^{-}$anions and two water molecules in the asymmetric unit. The crystal structure was solved by simulated annealing from laboratory $\mathrm{X}$-ray powder diffraction data, with data collected at room temperature. Rietveld refinement of this model led to a final $R_{\text {wp }}$ of 0.047 to $1.79 \AA$ resolution. A three-dimensional network of hydrogen bonding links the amodiaquinium cations via water molecules and $\mathrm{Cl}^{-}$ions.

\section{Comment}

Amodiaquine, 4-[(7-chloro-4-quinolinyl)amino]-2-[(diethylamino)methyl]phenol, is an antimalarial drug (Olliaro \& Mussano, 2003), often formulated as a dihydrochloride salt. This salt is known to exist in anhydrous, monohydrate and dihydrate forms. The crystal structure of the monohydrate form has been reported by Yennawar \& Viswamitra (1991). Here, the crystal structure of the dihydrate form, (I), is reported and compared with that of the monohydrate.<smiles>CC[NH+](CC)Cc1cc(Nc2cc[nH+]c3cc(Cl)ccc23)ccc1O</smiles>

(I)

The asymmetric unit of the dihydrate structure is shown in Fig. 1. It was assumed that the same $\mathrm{N}$ atoms were protonated on hydrochloride salt formation as in the monohydrate form (see Experimental). Yennawar \& Viswamitra (1991) found that the bonds that link the benzene and quinoline units through an $\mathrm{N}$ atom show strong double-bond character, as indicated by short $\mathrm{C}-\mathrm{N}$ bond lengths and close-to-planar $\mathrm{C}-$ $\mathrm{C}-\mathrm{N}-\mathrm{C}$ torsion angles (Table 2). A search of the Cambridge Structural Database (CSD, Version 5.27; Allen, 2002) was performed in order to determine typical values for these parameters. The average $\mathrm{C}_{\mathrm{ar}}-\mathrm{NH}$ bond length was found to be ca 1.39 (3) $\AA$ and the distribution of the $\mathrm{C}-\mathrm{C}-\mathrm{N}-\mathrm{C}$ torsion angles showed sharp maxima at $0^{\circ}$ and $180^{\circ}$. 


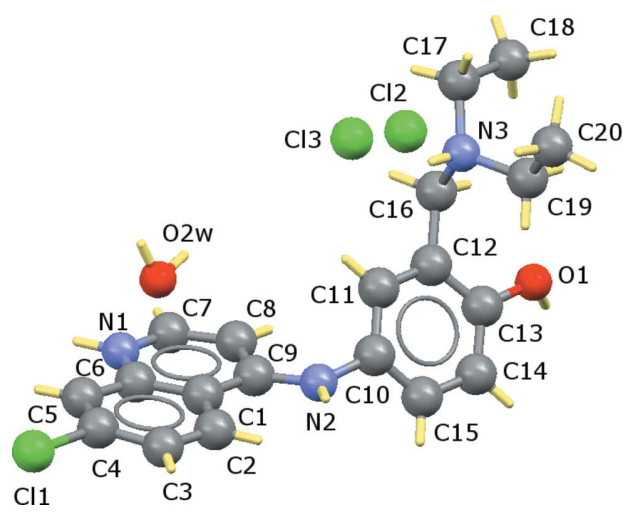

Figure 1

A view of (I), with the atom-numbering scheme.

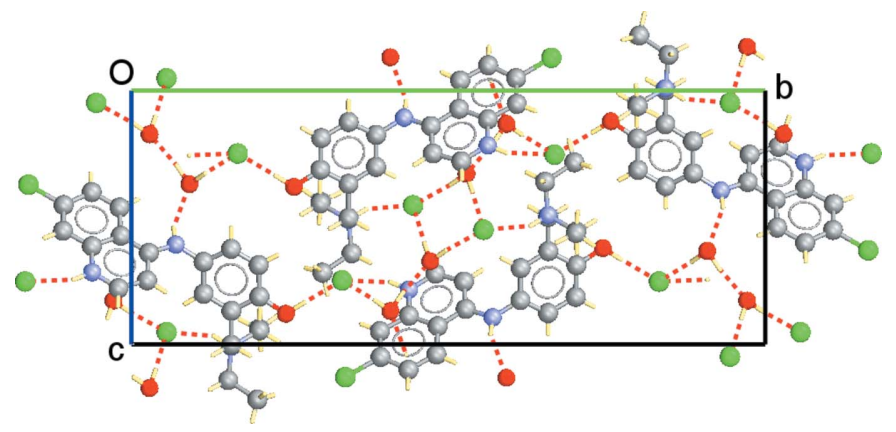

Figure 2

The crystal packing of (I), viewed along the $a$ axis. Hydrogen bonds are indicated by dashed lines.

In the dihydrate form, the $\mathrm{C} 8-\mathrm{C} 9-\mathrm{N} 2-\mathrm{C} 10$ torsion angle is closer to the ideal value of $0^{\circ}$ than in the monohydrate form, which suggests an increased $\mathrm{C}-\mathrm{N}$ double-bond character (Table 1). Even though the resolution of our data does not permit the determination of $\mathrm{H}$-atom positions, the coordinates of both $\mathrm{H} 2$ and $\mathrm{H} 2 \mathrm{~N}$ can reasonably be estimated using idealized bond geometry and normalized bond lengths. The distance between the calculated $\mathrm{H} 2$ and $\mathrm{H} 2 \mathrm{~N}$ positions is $1.85 \AA$ in the dihydrate, while $2.08 \AA$ was reported for the monohydrate. Thus, the $\mathrm{C} 8-\mathrm{C} 9-\mathrm{N} 2-\mathrm{C} 10$ torsion angle in the dihydrate form approaches planarity despite considerable steric congestion. This behaviour is indicative of strong C9$\mathrm{N} 2$ double-bond character.

The $\mathrm{C} 9-\mathrm{N} 2-\mathrm{C} 10-\mathrm{C} 11$ torsion angles are far from planar in both forms (Table 1). The coplanarity of the quinoline and benzene rings is sterically hindered by the close approach of the $\mathrm{C} 8-\mathrm{H} 8$ and the $\mathrm{C} 11-\mathrm{H} 11 \mathrm{H}$ atoms (Fig. 1).

The most apparent difference between the conformations of amodiaquine in the two structures is a rotation around the C12-C16 bond (Table 1), which moves the diethylamino group to opposite sides (above/below; see Fig. 1) of the benzene ring. In the dihydrate structure, one of the $\mathrm{H}$ atoms attached to atom $\mathrm{C} 16$ is involved in a close intramolecular contact of $2.41 \AA$ with atom $\mathrm{O} 1$.

The amodiaquinium cations donate hydrogen bonds to two $\mathrm{Cl}^{-}$ions and a water molecule in both forms (Table 2;

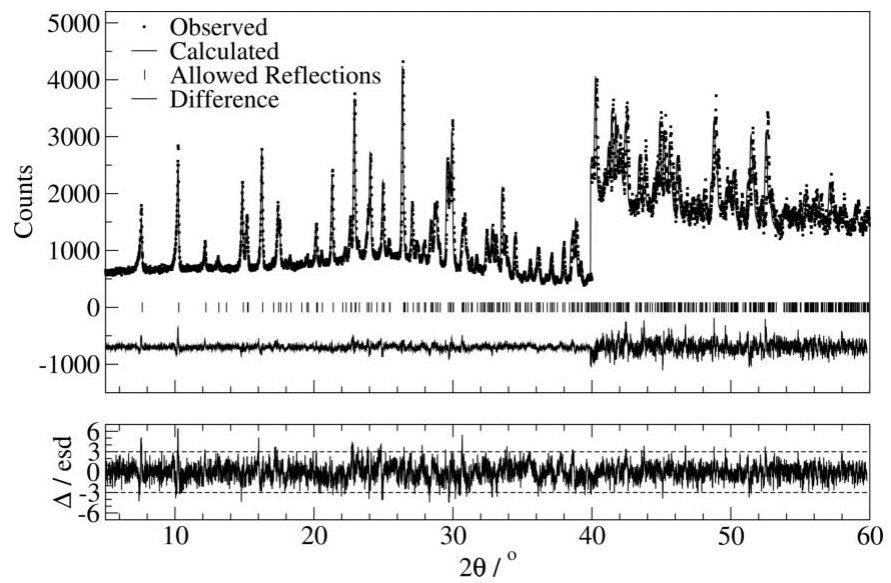

Figure 3

Final observed (points), calculated (line), difference $\left[\left(y_{\text {obs }}-y_{\text {calc }}\right)\right]$ and weighted difference $\left[\left(y_{\text {obs }}-y_{\text {calc }}\right) / \sigma\right]$ profiles for the Rietveld refinement of the title compound. Change of scale at $40^{\circ}$ is a factor of 10 and the increment in $2 \theta$ is $0.01^{\circ}$.

Yennawar \& Viswamitra, 1991). The roles of the donor groups, however, differ in the two forms. In the dihydrate, N2$\mathrm{H} 2 \mathrm{~N} \cdot \mathrm{OH}_{2}$ and $\mathrm{O} 1-\mathrm{H} 1 \mathrm{O} \cdots \mathrm{Cl}^{-}$hydrogen bonds are formed, while in the monohydrate there are $\mathrm{N}-\mathrm{H} \cdots \mathrm{Cl}^{-}$and $\mathrm{O}-\mathrm{H} \cdots \mathrm{OH}_{2}$ bonds.

Stacking interactions between the phenol and quinoline rings of screw-related molecules were found in the monohydrate structure (Yennawar \& Viswamitra, 1991). No such interactions are present in the crystal structure of the dihydrate form (Fig. 2). The monohydrate structure appears to be deficient in hydrogen-bond donors, since one of the $\mathrm{Cl}^{-}$ ions accepts only one hydrogen bond instead of the usual two or three (Infantes \& Motherwell, 2004). The additional donating ability of the extra water molecule in the dihydrate form permits a more optimal hydrogen-bonding scheme, and the stacking interactions between amodiaquinium cations are replaced by indirect hydrogen-bonded links through the solvent molecules and the counterions.

\section{Experimental}

Amodiaquinium dihydrochloride dihydrate was obtained from Sigma and used without further purification. No impurities were detected by $\mathrm{X}$-ray powder diffraction. The sample was ground lightly and loaded into a $0.7 \mathrm{~mm}$-diameter Lindemann glass capillary. Data were collected at room temperature and pressure in Debye-Scherrer geometry employing $\mathrm{Co} K \alpha_{1}$ radiation.

Crystal data

$\mathrm{C}_{20} \mathrm{H}_{24} \mathrm{ClN}_{3} \mathrm{O}^{2+} \cdot 2 \mathrm{Cl}^{-} \cdot 2 \mathrm{H}_{2} \mathrm{O}$
$M r=464.8$
Monoclinic, $P{ }_{1} / c$
$a=7.83868(10) \AA$
$b=26.9917(5) \AA$
$c=10.80804(18) \AA$
$\beta=92.9632(13)^{\circ}$
$V=2283.6(2) \AA^{3}$
$Z=4$
$D_{x}=1.352 \mathrm{Mg} \mathrm{m}^{-3}$

Co $K \alpha_{1}$ radiation $\mu=0 \mathrm{~mm}^{-1}$ $T=298 \mathrm{~K}$

Specimen shape: cylinder $12 \times 0.7 \times 0.7 \mathrm{~mm}$ Specimen prepared at $100 \mathrm{kPa}$ Specimen prepared at $298 \mathrm{~K}$ Particle morphology: fine powder, yellow 


\section{Data collection}

Stoe linear PSD diffractometer

Specimen mounting: $0.7 \mathrm{~mm}$

Lindemann glass capillary

Specimen mounted in transmission mode

\section{Refinement}

Refinement on $I_{\text {net }}$

$R_{\mathrm{p}}=0.037$

$R_{\mathrm{wp}}=0.047$

$R_{\exp }=0.038$

$R_{\mathrm{B}}=0.0622$

$S=1.29$

Wavelength of incident radiation: $1.78892 \AA$

Excluded region(s): none

Profile function: pseudo-Voigt

(Thompson et al., 1987) with

asymmetry correction (Finger et al., 1994)

392 reflections

181 parameters
Scan method: step Absorption correction: none

$2 \theta_{\text {min }}=2.0,2 \theta_{\max }=60.0^{\circ}$

Increment in $2 \theta=0.01^{\circ}$

$\mathrm{H}$ atoms treated by a mixture of independent and constrained refinement

Weighting scheme based on

measured s.u.'s,

$1 / \sigma\left(y_{\text {obs }}\right)^{2}$

$(\Delta / \sigma)_{\max }=0.14$

Preferred orientation correction:

March-Dollase, as implemented and documented in GSAS (Larson \& Von Dreele, 2000),

along the (100 axis),

ratio $=1.066(2)$,

range: $\min =0.82398$, $\max =1.10164$

\section{Table 1}

Selected geometric parameters $\left({ }^{\circ}\right)$ of amodiaquine in the crystal structures of amodiaquinium dihydrochloride monohydrate (CSD refcode VOTFIT; Yennawar \& Viswamitra, 1991) and amodiaquinium dihydrochloride dihydrate, (I). Atom labels are given in Fig. 1.

\begin{tabular}{llr}
\hline Parameter & Monohydrate & Dihydrate \\
\hline $\mathrm{C} 8-\mathrm{C} 9-\mathrm{N} 2-\mathrm{C} 10$ & $17.2(7)$ & $5.0(12)$ \\
$\mathrm{C} 9-\mathrm{N} 2-\mathrm{C} 10-\mathrm{C} 11$ & $34.7(7)$ & $46.9(12)$ \\
Quinoline-phenyl interplanar angle & 48 & $54.6(4)$ \\
C11-C12-C16-N3 & -91.4 & $77.4(8)$ \\
\hline
\end{tabular}

Table 2

Hydrogen-bond geometry $\left(\AA{ }^{\circ}\right)$.

\begin{tabular}{lllll}
\hline$D-\mathrm{H} \cdots A$ & $D-\mathrm{H}$ & $\mathrm{H} \cdots A$ & $D \cdots A$ & $D-\mathrm{H} \cdots A$ \\
\hline $\mathrm{N} 1-\mathrm{H} 1 \mathrm{~N} \cdots \mathrm{Cl} 2^{\mathrm{i}}$ & $0.93(1)$ & $2.26(1)$ & $3.160(6)$ & $162(1)$ \\
$\mathrm{N} 2-\mathrm{H} 2 \mathrm{~N} \cdots \mathrm{O} 2 W^{\text {ii }}$ & $0.93(2)$ & $2.07(2)$ & $2.902(12)$ & $148(1)$ \\
$\mathrm{N} 3-\mathrm{H} 3 \mathrm{~N} \cdots \mathrm{Cl} 3$ & $0.93(1)$ & $2.15(1)$ & $3.016(7)$ & $155(1)$ \\
$\mathrm{O} 1-\mathrm{H} 1 \mathrm{O} \cdots \mathrm{Cl} 2^{\text {iii }}$ & 0.98 & 2.06 & $3.037(7)$ & 177 \\
$\mathrm{O} 1 W-\mathrm{H} 1 W A \cdots \mathrm{Cl} 3$ & 0.97 & 2.16 & $3.127(11)$ & 175 \\
$\mathrm{O} 1 W-\mathrm{H} 1 W B \cdots \mathrm{Cl} 3^{\text {iv }}$ & 1.02 & 2.28 & $3.297(11)$ & 177 \\
$\mathrm{O} 2 W-\mathrm{H} 2 W A \cdots \mathrm{O} 1 W$ & 0.98 & 1.84 & $2.816(14)$ & 171 \\
$\mathrm{O} 2 W-\mathrm{H} 2 W B \cdots \mathrm{Cl} 2^{\text {iv }}$ & 1.06 & 2.13 & $3.188(12)$ & 177
\end{tabular}

Symmetry codes: (i) $-x+2,-y+1,-z+1$; $\quad$ (ii) $-x+1,-y+1,-z+2$; (iii) $x,-y+\frac{3}{2}, z+\frac{1}{2}$; (iv) $-x+1,-y+1,-z+1$.

The program DASH (David et al., 2004) was employed for structure solution. The powder pattern was truncated to $48.35^{\circ}$ in $2 \theta$ (Co $\mathrm{K} \alpha$ ), corresponding to a real-space resolution of $2.2 \AA$. The background was subtracted with a Bayesian high-pass filter (David \& Sivia, 2001). Peak positions for indexing were obtained by fitting with an asymmetry-corrected Voigt function (Thompson et al., 1987; Finger et al., 1994). Indexing with the program DICVOL91 (Boultif \& Louër, 1991) failed, but the same 24 peak positions could be indexed with the program DICVOL04 (Boultif \& Louër, 2004) without allowing for impurity peaks. Pawley refinement was used to extract integrated intensities and their correlations, from which the space

group was determined using Bayesian statistical analysis (Markvardsen et al., 2001). $P 2_{1} / c$ was returned as the only possible space group, which resulted in a Pawley $\chi^{2}$ of 0.70 . Simulated annealing was used to solve the crystal structure of compound (I) from the powder pattern in direct space. The starting molecular geometry was taken from the crystal structure of amodiaquinium dihydrochloride monohydrate (Yennawar \& Viswamitra, 1991) from the CSD (refcode VOTFIT). The molecule was assumed to be a salt in the solid state, based on the single-crystal structure of the monohydrate, where the two $\mathrm{H}$ atoms on the two positively charged $\mathrm{N}$ atoms had been located from the difference Fourier map. We note, however, that the three $\mathrm{H}$ atoms that were located only render two of the $\mathrm{N}$ atoms positive; charge balance therefore requires the hydroxide counterion that is stated to be present to be a water molecule. The structure of VOTFIT is therefore the monohydrate, and this has now been corrected in the CSD.

Because $\mathrm{H}$ atoms do not contribute significantly to the powder diffraction pattern, due to their low X-ray scattering power, they can be ignored during the structure solution process. Hence, a water molecule can be reduced to an $\mathrm{O}$ atom, which reduces its number of degrees of freedom from six to three. The amodiaquine molecule has six flexible torsion angles, which, combined with the two water molecules and the two $\mathrm{Cl}^{-}$ions, give a total of 24 degrees of freedom. Because of the large number of degrees of freedom, it cannot be expected that the default settings for simulated annealing in $D A S H$ (ten simulated annealing runs of 10000000 moves each) would be sufficient. Instead, 50 simulated annealing runs of 100000000 moves each were performed. In 50 simulated annealing runs, the correct crystal structure was found ten times, with a profile $\chi^{2}$ of $1.60,2.3$ times the Pawley $\chi^{2}$. The next best solution had a significantly higher profile $\chi^{2}$ of 7.66 .

The background subtraction, peak fitting, Pawley refinement, space-group determination and simulated-annealing algorithms were used as implemented in the program $D A S H$.

Rietveld refinement was carried out on the solution with the lowest profile $\chi^{2}$, with $\mathrm{H}$ atoms added in calculated positions. Bond lengths and angles involving heavy atoms were restrained to values taken from CSD entry VOTFIT (Yennawar \& Viswamitra, 1991). Planar group restraints were applied for aromatic rings. The $\mathrm{CH}, \mathrm{CH}_{2}$ and $\mathrm{CH}_{3}$ distances were restrained to be $0.93(1), 0.97$ (1) and 0.96 (1) $\AA$ respectively, with idealized bond angles. The refinement (Fig. 3), using the GSAS software suite (Larson \& Von Dreele, 2000), converged readily to yield acceptable figures of merit $\left(\chi^{2}=1.655, R_{\mathrm{p}}\right.$ $\left.=0.0366, R_{\mathrm{wp}}=0.0465\right)$ and a chemically reasonable structural model. A single overall isotropic displacement parameter was employed. The orientations of the water molecules were kept fixed to enforce a chemically reasonable hydrogen-bonding geometry. Reported standard deviations are taken from the program employed and represent statistical uncertainties rather than estimates of the absolute error, which are likely to be considerably greater.

Data collection: WinXPow (Stoe, 1999); cell refinement: GSAS (Larson \& Von Dreele, 2000); data reduction: WinXPow; program(s) used to solve structure: DASH (David et al., 2004); program(s) used to refine structure: GSAS; molecular graphics: MERCURY (Macrae et al., 2006); software used to prepare material for publication: $v i$ (http://www.vim.org/).

AL, LF and JMG thank the Pfizer Institute for Pharmaceutical Materials Science for funding. JB thanks Jesus College, Cambridge, for the award of a Junior Research Fellowship. 


\section{References}

Allen, F. H. (2002). Acta Cryst. B58, 380-388.

Boultif, A. \& Louër, D. (1991). J. Appl. Cryst. 24, 987-993.

Boultif, A. \& Louër, D. (2004). J. Appl. Cryst. 37, 724-731.

David, W. I. F., Shankland, K., van de Streek, J., Pidcock, E. \& Motherwell, S.

(2004). DASH. Version 3.0. Cambridge Crystallographic Data Centre, 12 Union Road, Cambridge, England.

David, W. I. F. \& Sivia, D. S. (2001). J. Appl. Cryst. 34, 318-324.

Finger, L. W., Cox, D. E. \& Jephcoat, A. P. (1994). J. Appl. Cryst. 27, 892-900.

Infantes, L. \& Motherwell, W. D. S. (2004). Chem. Commun. pp. 1166-1167.
Larson, A. C. \& Von Dreele, R. B. (2000). GSAS. Report LAUR 86-748. Los Alamos National Laboratory, New Mexico, USA.

Macrae, C. F., Edgington, P. R., McCabe, P., Pidcock, E., Shields, G. P., Taylor, R., Towler, M. \& van de Streek, J. (2006). J. Appl. Cryst. 39, 453-457.

Markvardsen, A. J., David, W. I. F., Johnson, J. C. \& Shankland, K. (2001). Acta Cryst. A57, 47-54.

Olliaro, P. \& Mussano, P. (2003). Amodiaquine for Treating Malaria in The Cochrane Database of Systematic Reviews. (http://www.cochrane.org/ reviews/index.htm)

Stoe (1999). WinXPow. Stoe \& Cie, Darmstadt, Germany.

Thompson, P., Cox, D. E. \& Hastings, J. B. (1987). J. Appl. Cryst. 20, 79-83.

Yennawar, H. P. \& Viswamitra, M. A. (1991). Curr. Sci. 61, 39-43. 


\section{supporting information}

Acta Cryst. (2006). E62, o4196-o4199 [https://doi.org/10.1107/S1600536806033691]

\section{Amodiaquinium dichloride dihydrate from laboratory powder diffraction data}

Antonio Llinàs, László Fábián, Jonathan C. Burley, Jacco van de Streek and Jonathan M.

\section{Goodman}

5-[(7-chloroquinolinium-4-yl)amino]-2-hydroxybenzyl\}dimethylammonium dichloride dihydrate

Crystal data

$\mathrm{C}_{20} \mathrm{H}_{24} \mathrm{ClN}_{3} \mathrm{O}^{2+} \cdot 2 \mathrm{Cl}^{-} \cdot 2 \mathrm{H}_{2} \mathrm{O}$

$M_{r}=464.8$

Monoclinic, $P 2_{1} / c$

Hall symbol: -P $2 \mathrm{ybc}$

$a=7.83868(10) \AA$

$b=26.9917(5) \AA$

$c=10.80804$ (18) $\AA$

$\beta=92.9632(13)^{\circ}$

$V=2283.6(2) \AA^{3}$

$Z=4$

\section{Data collection}

Stoe linear PSD diffractometer

Radiation source: sealed X-ray tube, Stoe STADI-P

Primary focusing, Ge 111 monochromator

Refinement

Refinement on $I_{\text {net }}$

Least-squares matrix: selected elements only

$R_{\mathrm{p}}=0.037$

$R_{\text {wp }}=0.047$

$R_{\text {exp }}=0.038$

$R\left(F^{2}\right)=0.05179$

7199 data points

Profile function: pseudo-Voigt (Thompson et al., 1987) with asymmetry correction (Finger et al., 1994)

181 parameters

164 restraints
$F(000)=976$

$D_{\mathrm{x}}=1.352 \mathrm{Mg} \mathrm{m}^{-3}$

Co $K \alpha_{1}$ radiation, $\lambda=1.78892 \AA$

$\mu=0 \mathrm{~mm}^{-1}$

$T=298 \mathrm{~K}$

Particle morphology: Please complete yellow

cylinder, $12 \times 0.7 \mathrm{~mm}$

Specimen preparation: Prepared at $298 \mathrm{~K}$

Specimen mounting: $0.7 \mathrm{~mm}$ Lindemann glass capillary

Data collection mode: transmission

Scan method: step

$2 \theta_{\min }=2.0^{\circ}, 2 \theta_{\max }=79.99^{\circ}, 2 \theta_{\text {step }}=0.01^{\circ}$

$\mathrm{H}$ atoms treated by a mixture of independent and constrained refinement

Weighting scheme based on measured s.u.'s $1 / \sigma\left(\mathrm{Y}_{\mathrm{obs}}\right)^{2}$

$(\Delta / \sigma)_{\max }=0.14$

Background function: Shifted Chebyshev polynomial of the first type, 15 terms, GSAS ((Larson \& Von Dreele, 2000).

Preferred orientation correction: MarchDollase, as implemented and documented in GSAS (Larson \& Von Dreele, 2000), along the $(100$ axis), ratio $=1.066(2)$, Prefered orientation correction range: $\min =0.82398, \max =1.10164$ 
Special details

Refinement. CW Profile function number 3 with 19 terms Pseudo-Voigt profile coefficients as parameterized in P. Thompson, D·E. Cox \& J·B. Hastings (1987). J. Appl. Cryst.,20,79-83. Asymmetry correction of L·W. Finger, D·E. Cox \& A. P. Jephcoat (1994). J. Appl. Cryst.,27,892-900. \#1(GU) $=133.258 \# 2(\mathrm{GV})=0.000 \# 3(\mathrm{GW})=4.899 \# 4(\mathrm{GP})=0.000$ $\# 5(\mathrm{LX})=4.575 \# 6(\mathrm{LY})=0.000 \# 7(S / L)=0.0130 \# 8(\mathrm{H} / L)=0.0350 \# 9(\operatorname{trns})=0.00 \# 10(\mathrm{shft})=0.0000 \# 11(\mathrm{stec})=0.00$ $\# 12($ ptec $)=0.00 \# 13(\mathrm{sfec})=0.00 \# 14(\mathrm{~L} 11)=0.000 \# 15(\mathrm{~L} 22)=0.000 \# 16(\mathrm{~L} 33)=0.000 \# 17(\mathrm{~L} 12)=0.000 \# 18(\mathrm{~L} 13)=$ $0.000 \# 19(\mathrm{~L} 23)=0.000$ Peak tails are ignored where the intensity is below 0.0010 times the peak

Fractional atomic coordinates and isotropic or equivalent isotropic displacement parameters $\left(\hat{A}^{2}\right)$

\begin{tabular}{|c|c|c|c|c|}
\hline & $x$ & $y$ & $z$ & $U_{\text {iso }} * / U_{\text {eq }}$ \\
\hline $\mathrm{C} 1$ & $0.7990(14)$ & $0.48320(19)$ & $0.9281(7)$ & $0.1333(14)^{*}$ \\
\hline $\mathrm{C} 2$ & $0.7130(15)$ & $0.48123(2)$ & $1.0395(8)$ & $0.1333(14)^{*}$ \\
\hline $\mathrm{H} 2$ & $0.6666(3)$ & $0.50990(2)$ & $1.0716(14)$ & $0.1333(14)^{*}$ \\
\hline $\mathrm{C} 3$ & $0.6969(13)$ & $0.43719(18)$ & $1.0998(7)$ & $0.1333(14)^{*}$ \\
\hline H3 & $0.6373(2)$ & $0.43588(2)$ & $1.1719(11)$ & $0.1333(14)^{*}$ \\
\hline $\mathrm{C} 4$ & $0.7618(13)$ & $0.39305(17)$ & $1.0494(7)$ & $0.1333(14)^{*}$ \\
\hline $\mathrm{Cl1}$ & $0.7399(6)$ & $0.33753(16)$ & $1.1292(4)$ & $0.1333(14)^{*}$ \\
\hline $\mathrm{C} 5$ & $0.8521(12)$ & $0.39204(18)$ & $0.9475(7)$ & $0.1333(14)^{*}$ \\
\hline H5 & $0.9022(16)$ & $0.36312(2)$ & $0.9200(9)$ & $0.1333(14)^{*}$ \\
\hline C6 & $0.8712(11)$ & 0.43766 (19) & $0.8871(7)$ & $0.1333(14)^{*}$ \\
\hline N1 & $0.9654(10)$ & $0.43801(2)$ & $0.7841(6)$ & $0.1333(14)^{*}$ \\
\hline $\mathrm{H} 1 \mathrm{~N}$ & $1.0109(17)$ & $0.40865(3)$ & $0.7551(10)$ & $0.1333(14)^{*}$ \\
\hline $\mathrm{C} 7$ & $0.9826(12)$ & $0.47951(3)$ & $0.7216(7)$ & $0.1333(14)^{*}$ \\
\hline $\mathrm{H} 7$ & $1.0455(2)$ & $0.47865(3)$ & $0.6511(12)$ & $0.1333(14)^{*}$ \\
\hline $\mathrm{C} 8$ & $0.9044(10)$ & $0.52451(3)$ & $0.7524(6)$ & $0.1333(14)^{*}$ \\
\hline $\mathrm{H} 8$ & $0.9262(19)$ & 0.55311 & $0.7079(10)$ & $0.1333(14)^{*}$ \\
\hline C9 & $0.8183(11)$ & $0.52747(2)$ & $0.8605(7)$ & $0.1333(14)^{*}$ \\
\hline $\mathrm{N} 2$ & $0.7370(10)$ & $0.56989(2)$ & $0.8933(5)$ & $0.1333(14)^{*}$ \\
\hline $\mathrm{H} 2 \mathrm{~N}$ & $0.6669(3)$ & $0.5666(4)$ & $0.9597(16)$ & $0.1333(14)^{*}$ \\
\hline $\mathrm{C} 10$ & $0.7152(9)$ & $0.61374(18)$ & $0.8255(5)$ & $0.1333(14)^{*}$ \\
\hline C11 & $0.6597(14)$ & $0.61408(15)$ & $0.6994(5)$ & $0.1333(14)^{*}$ \\
\hline H11 & $0.6230(3)$ & $0.58484(2)$ & $0.6610(7)$ & $0.1333(14)^{*}$ \\
\hline $\mathrm{C} 12$ & $0.6600(10)$ & $0.65765(13)$ & $0.6326(4)$ & $0.1333(14)^{*}$ \\
\hline $\mathrm{C} 13$ & $0.7070(13)$ & $0.70191(15)$ & $0.6884(4)$ & $0.1333(14)^{*}$ \\
\hline $\mathrm{O} 1$ & $0.7076(11)$ & $0.74355(15)$ & $0.6207(5)$ & $0.1333(14)^{*}$ \\
\hline $\mathrm{H} 1 \mathrm{O}$ & 0.75705 & 0.77244 & 0.66446 & $0.1333(14)^{*}$ \\
\hline C14 & $0.7715(15)$ & $0.70075(2)$ & $0.8127(5)$ & $0.1333(14)^{*}$ \\
\hline H14 & $0.8095(3)$ & $0.72981(3)$ & $0.8512(8)$ & $0.1333(14)^{*}$ \\
\hline $\mathrm{C} 15$ & $0.7790(12)$ & $0.65725(2)$ & $0.8762(5)$ & $0.1333(14)^{*}$ \\
\hline H15 & $0.8188(2)$ & $0.65746(3)$ & $0.9588(7)$ & $0.1333(14)^{*}$ \\
\hline C16 & $0.5877(8)$ & $0.65740(19)$ & $0.4983(4)$ & $0.1333(14)^{*}$ \\
\hline H16A & $0.6280(10)$ & $0.62802(3)$ & $0.4572(6)$ & $0.1333(14)^{*}$ \\
\hline H16B & $0.6284(9)$ & $0.68653(3)$ & $0.4564(6)$ & $0.1333(14)^{*}$ \\
\hline N3 & $0.3910(8)$ & $0.65751(16)$ & $0.4907(6)$ & $0.1333(14)^{*}$ \\
\hline $\mathrm{H} 3 \mathrm{~N}$ & $0.3550(10)$ & $0.62970(2)$ & $0.5329(8)$ & $0.1333(14)^{*}$ \\
\hline $\mathrm{C} 17$ & $0.3268(10)$ & $0.65258(3)$ & $0.3591(7)$ & $0.1333(14)^{*}$ \\
\hline H17A & $0.2042(12)$ & $0.6474(6)$ & $0.3567(10)$ & $0.1333(14)^{*}$ \\
\hline
\end{tabular}




$\begin{array}{lllll}\text { H17B } & 0.3807(2) & 0.6241(4) & 0.3227(9) & 0.1333(14)^{*} \\ \text { C18 } & 0.3649(15) & 0.6987(4) & 0.2829(6) & 0.1333(14)^{*} \\ \text { H18A } & 0.478(6) & 0.7101(3) & 0.305(8) & 0.1333(14)^{*} \\ \text { H18B } & 0.356(15) & 0.6905(13) & 0.1963(7) & 0.1333(14)^{*} \\ \text { H18C } & 0.284(9) & 0.7242(14) & 0.300(9) & 0.1333(14)^{*} \\ \text { C19 } & 0.3271(9) & 0.70192(3) & 0.5556(8) & 0.1333(14)^{*} \\ \text { H19A } & 0.3609(17) & 0.73147(2) & 0.5120(16) & 0.1333(14)^{*} \\ \text { H19B } & 0.3778(14) & 0.7027(6) & 0.6394(10) & 0.1333(14)^{*} \\ \text { C20 } & 0.1324(9) & 0.7013(4) & 0.5611(12) & 0.1333(14)^{*} \\ \text { H20A } & 0.0973(16) & 0.6709(18) & 0.598(10) & 0.1333(14)^{*} \\ \text { H20B } & 0.0966(13) & 0.7289(2) & 0.610(9) & 0.1333(14)^{*} \\ \text { H20C } & 0.0812(14) & 0.704(4) & 0.4786(17) & 0.1333(14)^{*} \\ \text { C12 } & 0.8709(6) & 0.66762(18) & 0.2523(4) & 0.1333(14)^{*} \\ \text { C13 } & 0.2586(6) & 0.55560(17) & 0.5482(4) & 0.1333(14)^{*} \\ \text { O1W } & 0.4815(12) & 0.4714(4) & 0.6724(9) & 0.1333(14)^{*} \\ \text { H1WA } & 0.41075 & 0.49619 & 0.62992 & 0.1333(14)^{*} \\ \text { H1WB } & 0.55814 & 0.46234 & 0.60282 & 0.1333(14)^{*} \\ \text { O2W } & 0.4008(14) & 0.4084(4) & 0.8686(9) & 0.1333(14)^{*} \\ \text { H2WA } & 0.42048 & 0.42866 & 0.79513 & 0.1333(14)^{*} \\ \text { H2WB } & 0.30932 & 0.38409 & 0.82629 & 0.1333(14)^{*} \\ & & & & \end{array}$

Geometric parameters $\left(\AA,{ }^{\circ}\right)$

\begin{tabular}{llll}
\hline $\mathrm{C} 1-\mathrm{C} 2$ & $1.4108(17)$ & $\mathrm{C} 14-\mathrm{H} 14$ & $0.9299(18)$ \\
$\mathrm{C} 1-\mathrm{C} 6$ & $1.4329(16)$ & $\mathrm{C} 14-\mathrm{C} 15$ & $1.3597(17)$ \\
$\mathrm{C} 1-\mathrm{C} 9$ & $1.4129(17)$ & $\mathrm{H} 14-\mathrm{C} 14$ & $0.9299(18)$ \\
$\mathrm{C} 2-\mathrm{H} 2$ & $0.9299(18)$ & $\mathrm{C} 15-\mathrm{C} 10$ & $1.3792(17)$ \\
$\mathrm{C} 2-\mathrm{C} 3$ & $1.3645(17)$ & $\mathrm{C} 15-\mathrm{C} 14$ & $1.3597(17)$ \\
$\mathrm{H} 2-\mathrm{C} 2$ & $0.9299(18)$ & $\mathrm{C} 15-\mathrm{H} 15$ & $0.9301(18)$ \\
$\mathrm{C} 3-\mathrm{H} 3$ & $0.9300(18)$ & $\mathrm{H} 15-\mathrm{C} 15$ & $0.9301(18)$ \\
$\mathrm{C} 3-\mathrm{C} 4$ & $1.4153(17)$ & $\mathrm{C} 16-\mathrm{C} 12$ & $1.5300(18)$ \\
$\mathrm{H} 3-\mathrm{C} 3$ & $0.9300(18)$ & $\mathrm{C} 16-\mathrm{H} 16 \mathrm{~A}$ & $0.9700(18)$ \\
$\mathrm{C} 4-\mathrm{C} 5$ & $1.3397(17)$ & $\mathrm{C} 16-\mathrm{H} 16 \mathrm{~B}$ & $0.9699(18)$ \\
$\mathrm{C} 5-\mathrm{C} 4$ & $1.3397(17)$ & $\mathrm{C} 16-\mathrm{N} 3$ & $1.5398(18)$ \\
$\mathrm{C} 5-\mathrm{H} 5$ & $0.9299(18)$ & $\mathrm{H} 16 \mathrm{~A}-\mathrm{C} 16$ & $0.9700(18)$ \\
$\mathrm{C} 5-\mathrm{C} 6$ & $1.4055(17)$ & $\mathrm{H} 16 \mathrm{~B}-\mathrm{C} 16$ & $0.9699(18)$ \\
$\mathrm{H} 5-\mathrm{C} 5$ & $0.9299(18)$ & $\mathrm{N} 3-\mathrm{C} 16$ & $1.5398(18)$ \\
$\mathrm{C} 6-\mathrm{C} 5$ & $1.4055(17)$ & $\mathrm{N} 3-\mathrm{H} 3 \mathrm{~N}$ & $0.9300(18)$ \\
$\mathrm{N} 1-\mathrm{C} 6$ & $1.3678(17)$ & $\mathrm{N} 3-\mathrm{C} 17$ & $1.4897(18)$ \\
$\mathrm{N} 1-\mathrm{H} 1 \mathrm{~N}$ & $0.9298(18)$ & $\mathrm{N} 3-\mathrm{C} 19$ & $1.4891(18)$ \\
$\mathrm{N} 1-\mathrm{C} 7$ & $1.3182(17)$ & $\mathrm{H} 3 \mathrm{~N}-\mathrm{N} 3$ & $0.9300(18)$ \\
$\mathrm{H} 1 \mathrm{~N}-\mathrm{N} 1$ & $0.9298(18)$ & $\mathrm{C} 17-\mathrm{N} 3$ & $1.4897(18)$ \\
$\mathrm{C} 7-\mathrm{N} 1$ & $1.3182(17)$ & $\mathrm{C} 17-\mathrm{H} 17 \mathrm{~A}$ & $0.9701(18)$ \\
$\mathrm{C} 7-\mathrm{H} 7$ & $0.9301(18)$ & $\mathrm{C} 17-\mathrm{H} 17 \mathrm{~B}$ & $0.9700(18)$ \\
$\mathrm{C} 7-\mathrm{C} 8$ & $1.4080(17)$ & $\mathrm{C} 17-\mathrm{C} 18$ & $1.5297(18)$ \\
$\mathrm{H} 7-\mathrm{C} 7$ & $0.9301(18)$ & $\mathrm{H} 17 \mathrm{~A}-\mathrm{C} 17$ & $0.9701(18)$ \\
$\mathrm{C} 8-\mathrm{C} 7$ & $1.4080(17)$ & $\mathrm{H} 17 \mathrm{~B}-\mathrm{C} 17$ & $0.9700(18)$ \\
$\mathrm{C} 8-\mathrm{H} 8$ & $0.9300(18)$ & $\mathrm{C} 18-\mathrm{C} 17$ & $1.5297(18)$ \\
& & &
\end{tabular}




\begin{tabular}{|c|c|c|c|}
\hline $\mathrm{C} 8-\mathrm{C} 9$ & $1.3811(17)$ & $\mathrm{C} 18-\mathrm{H} 18 \mathrm{~A}$ & $0.9600(18)$ \\
\hline $\mathrm{H} 8-\mathrm{C} 8$ & $0.9300(18)$ & $\mathrm{C} 18-\mathrm{H} 18 \mathrm{~B}$ & $0.9603(18)$ \\
\hline $\mathrm{C} 9-\mathrm{N} 2$ & $1.3664(18)$ & $\mathrm{C} 18-\mathrm{H} 18 \mathrm{C}$ & $0.9600(18)$ \\
\hline $\mathrm{N} 2-\mathrm{C} 9$ & $1.3664(18)$ & $\mathrm{H} 18 \mathrm{~A}-\mathrm{C} 18$ & $0.9600(18)$ \\
\hline $\mathrm{N} 2-\mathrm{H} 2 \mathrm{~N}$ & $0.9298(18)$ & $\mathrm{H} 18 \mathrm{~B}-\mathrm{C} 18$ & $0.9603(18)$ \\
\hline $\mathrm{N} 2-\mathrm{C} 10$ & $1.3981(18)$ & $\mathrm{H} 18 \mathrm{C}-\mathrm{C} 18$ & $0.9600(18)$ \\
\hline $\mathrm{H} 2 \mathrm{~N}-\mathrm{N} 2$ & $0.9298(18)$ & $\mathrm{C} 19-\mathrm{N} 3$ & $1.4891(18)$ \\
\hline $\mathrm{C} 10-\mathrm{N} 2$ & $1.3981(18)$ & C19-H19A & $0.9701(18)$ \\
\hline $\mathrm{C} 10-\mathrm{C} 11$ & $1.4092(17)$ & C19-H19B & $0.9702(18)$ \\
\hline $\mathrm{C} 10-\mathrm{C} 15$ & $1.3792(17)$ & $\mathrm{C} 19-\mathrm{C} 20$ & $1.5302(18)$ \\
\hline $\mathrm{C} 11-\mathrm{H} 11$ & $0.9301(18)$ & $\mathrm{H} 19 \mathrm{~A}-\mathrm{C} 19$ & $0.9701(18)$ \\
\hline $\mathrm{C} 11-\mathrm{C} 12$ & $1.3800(17)$ & $\mathrm{H} 19 \mathrm{~B}-\mathrm{C} 19$ & $0.9702(18)$ \\
\hline $\mathrm{H} 11-\mathrm{C} 11$ & $0.9301(18)$ & $\mathrm{C} 20-\mathrm{H} 20 \mathrm{~A}$ & $0.9604(18)$ \\
\hline $\mathrm{C} 12-\mathrm{C} 13$ & $1.3798(17)$ & $\mathrm{C} 20-\mathrm{H} 20 \mathrm{~B}$ & $0.9601(18)$ \\
\hline $\mathrm{C} 12-\mathrm{C} 16$ & $1.5300(18)$ & $\mathrm{C} 20-\mathrm{H} 20 \mathrm{C}$ & $0.9603(18)$ \\
\hline $\mathrm{C} 13-\mathrm{C} 14$ & $1.4110(17)$ & $\mathrm{O} 1 \mathrm{~W}-\mathrm{H} 1 \mathrm{WA}$ & $0.969(10)$ \\
\hline $\mathrm{O} 1-\mathrm{C} 13$ & $1.3412(18)$ & $\mathrm{O} 1 \mathrm{~W}-\mathrm{H} 1 \mathrm{WB}$ & $1.017(10)$ \\
\hline $\mathrm{O} 1-\mathrm{H} 1 \mathrm{O}$ & $0.9811(18)$ & $\mathrm{O} 2 \mathrm{~W}-\mathrm{H} 2 \mathrm{WA}$ & $0.983(9)$ \\
\hline $\mathrm{H} 1 \mathrm{O}-\mathrm{O} 1$ & $0.9811(18)$ & $\mathrm{O} 2 \mathrm{~W}-\mathrm{H} 2 \mathrm{WB}$ & $1.059(11)$ \\
\hline $\mathrm{C} 14-\mathrm{C} 13$ & $1.4110(17)$ & & \\
\hline $\mathrm{C} 2-\mathrm{C} 1-\mathrm{C} 6$ & $116.43(13)$ & $\mathrm{C} 13-\mathrm{C} 14-\mathrm{H} 14$ & $119.88(17)$ \\
\hline $\mathrm{C} 2-\mathrm{C} 1-\mathrm{C} 9$ & $122.84(13)$ & $\mathrm{C} 13-\mathrm{C} 14-\mathrm{C} 15$ & $120.26(12)$ \\
\hline $\mathrm{C} 6-\mathrm{C} 1-\mathrm{C} 9$ & $120.71(13)$ & $\mathrm{H} 14-\mathrm{C} 14-\mathrm{C} 15$ & $119.86(19)$ \\
\hline $\mathrm{C} 1-\mathrm{C} 2-\mathrm{H} 2$ & $120.01(18)$ & $\mathrm{C} 10-\mathrm{C} 15-\mathrm{C} 14$ & $121.96(16)$ \\
\hline $\mathrm{C} 1-\mathrm{C} 2-\mathrm{C} 3$ & $120.03(12)$ & $\mathrm{C} 10-\mathrm{C} 15-\mathrm{H} 15$ & $118.90(15)$ \\
\hline $\mathrm{H} 2-\mathrm{C} 2-\mathrm{C} 3$ & 119.96 (19) & $\mathrm{C} 14-\mathrm{C} 15-\mathrm{H} 15$ & $118.88(15)$ \\
\hline $\mathrm{C} 2-\mathrm{C} 3-\mathrm{H} 3$ & $119.75(15)$ & $\mathrm{C} 12-\mathrm{C} 16-\mathrm{H} 16 \mathrm{~A}$ & $109.00(16)$ \\
\hline $\mathrm{C} 2-\mathrm{C} 3-\mathrm{C} 4$ & $120.39(16)$ & $\mathrm{C} 12-\mathrm{C} 16-\mathrm{H} 16 \mathrm{~B}$ & $109.00(16)$ \\
\hline $\mathrm{H} 3-\mathrm{C} 3-\mathrm{C} 4$ & $119.76(15)$ & $\mathrm{C} 12-\mathrm{C} 16-\mathrm{N} 3$ & $111.81(16)$ \\
\hline $\mathrm{C} 3-\mathrm{C} 4-\mathrm{C} 5$ & $123.28(12)$ & $\mathrm{H} 16 \mathrm{~A}-\mathrm{C} 16-\mathrm{H} 16 \mathrm{~B}$ & $108.98(17)$ \\
\hline $\mathrm{C} 4-\mathrm{C} 5-\mathrm{H} 5$ & $121.99(17)$ & $\mathrm{H} 16 \mathrm{~A}-\mathrm{C} 16-\mathrm{N} 3$ & $109.00(17)$ \\
\hline $\mathrm{C} 4-\mathrm{C} 5-\mathrm{C} 6$ & $115.96(12)$ & $\mathrm{H} 16 \mathrm{~B}-\mathrm{C} 16-\mathrm{N} 3$ & $109.00(17)$ \\
\hline $\mathrm{H} 5-\mathrm{C} 5-\mathrm{C} 6$ & $122.01(15)$ & $\mathrm{C} 16-\mathrm{N} 3-\mathrm{H} 3 \mathrm{~N}$ & $107.47(17)$ \\
\hline $\mathrm{C} 1-\mathrm{C} 6-\mathrm{C} 5$ & $123.68(12)$ & $\mathrm{C} 16-\mathrm{N} 3-\mathrm{C} 17$ & $109.84(16)$ \\
\hline $\mathrm{C} 1-\mathrm{C} 6-\mathrm{N} 1$ & $118.98(11)$ & $\mathrm{C} 16-\mathrm{N} 3-\mathrm{C} 19$ & $109.68(16)$ \\
\hline $\mathrm{C} 5-\mathrm{C} 6-\mathrm{N} 1$ & $117.34(12)$ & $\mathrm{H} 3 \mathrm{~N}-\mathrm{N} 3-\mathrm{C} 17$ & $107.49(17)$ \\
\hline $\mathrm{C} 6-\mathrm{N} 1-\mathrm{H} 1 \mathrm{~N}$ & $120.07(14)$ & $\mathrm{H} 3 \mathrm{~N}-\mathrm{N} 3-\mathrm{C} 19$ & $107.48(16)$ \\
\hline $\mathrm{C} 6-\mathrm{N} 1-\mathrm{C} 7$ & $119.74(12)$ & $\mathrm{C} 17-\mathrm{N} 3-\mathrm{C} 19$ & $114.59(16)$ \\
\hline $\mathrm{H} 1 \mathrm{~N}-\mathrm{N} 1-\mathrm{C} 7$ & $120.04(19)$ & $\mathrm{N} 3-\mathrm{C} 17-\mathrm{H} 17 \mathrm{~A}$ & $108.92(17)$ \\
\hline $\mathrm{N} 1-\mathrm{C} 7-\mathrm{H} 7$ & $118.11(15)$ & N3-C17-H17B & $108.95(17)$ \\
\hline $\mathrm{N} 1-\mathrm{C} 7-\mathrm{C} 8$ & $123.73(12)$ & N3-C17-C18 & $112.07(16)$ \\
\hline $\mathrm{H} 7-\mathrm{C} 7-\mathrm{C} 8$ & $118.10(16)$ & $\mathrm{H} 17 \mathrm{~A}-\mathrm{C} 17-\mathrm{H} 17 \mathrm{~B}$ & $108.97(17)$ \\
\hline $\mathrm{C} 7-\mathrm{C} 8-\mathrm{H} 8$ & $120.01(15)$ & $\mathrm{H} 17 \mathrm{~A}-\mathrm{C} 17-\mathrm{C} 18$ & $108.93(17)$ \\
\hline $\mathrm{C} 7-\mathrm{C} 8-\mathrm{C} 9$ & $119.14(15)$ & $\mathrm{H} 17 \mathrm{~B}-\mathrm{C} 17-\mathrm{C} 18$ & $108.95(16)$ \\
\hline $\mathrm{H} 8-\mathrm{C} 8-\mathrm{C} 9$ & $120.0(2)$ & $\mathrm{C} 17-\mathrm{C} 18-\mathrm{H} 18 \mathrm{~A}$ & $109.47(2)$ \\
\hline $\mathrm{C} 1-\mathrm{C} 9-\mathrm{C} 8$ & $117.32(14)$ & $\mathrm{C} 17-\mathrm{C} 18-\mathrm{H} 18 \mathrm{~B}$ & $109.48(2)$ \\
\hline $\mathrm{C} 1-\mathrm{C} 9-\mathrm{N} 2$ & $120.66(13)$ & $\mathrm{C} 17-\mathrm{C} 18-\mathrm{H} 18 \mathrm{C}$ & $109.4(4)$ \\
\hline
\end{tabular}




$\begin{array}{ll}\mathrm{C} 8-\mathrm{C} 9-\mathrm{N} 2 & 121.55(16) \\ \mathrm{C} 9-\mathrm{N} 2-\mathrm{H} 2 \mathrm{~N} & 115.05(19) \\ \mathrm{C} 9-\mathrm{N} 2-\mathrm{C} 10 & 128.25(17) \\ \mathrm{H} 2 \mathrm{~N}-\mathrm{N} 2-\mathrm{C} 10 & 115.04(18) \\ \mathrm{N} 2-\mathrm{C} 10-\mathrm{C} 11 & 122.43(14) \\ \mathrm{N} 2-\mathrm{C} 10-\mathrm{C} 15 & 118.71(18) \\ \mathrm{C} 11-\mathrm{C} 10-\mathrm{C} 15 & 117.79(12) \\ \mathrm{C} 10-\mathrm{C} 11-\mathrm{H} 11 & 119.89(17) \\ \mathrm{C} 10-\mathrm{C} 11-\mathrm{C} 12 & 120.19(12) \\ \mathrm{H} 11-\mathrm{C} 11-\mathrm{C} 12 & 119.92(18) \\ \mathrm{C} 11-\mathrm{C} 12-\mathrm{C} 13 & 121.11(11) \\ \mathrm{C} 11-\mathrm{C} 12-\mathrm{C} 16 & 118.80(15) \\ \mathrm{C} 13-\mathrm{C} 12-\mathrm{C} 16 & 119.73(16) \\ \mathrm{C} 12-\mathrm{C} 13-\mathrm{O} 1 & 119.71(14) \\ \mathrm{C} 12-\mathrm{C} 13-\mathrm{C} 14 & 118.13(14) \\ \mathrm{O} 1-\mathrm{C} 13-\mathrm{C} 14 & 121.77(17) \\ \mathrm{C} 13-\mathrm{O} 1-\mathrm{H} 1 \mathrm{O} & 114.6(4)\end{array}$

$\begin{array}{ll}\text { H18A-C18-H18B } & 109.47(17) \\ \text { H18A-C18-H18C } & 109.48(2) \\ \text { H18B-C18-H18C } & 109.48(2) \\ \text { N3-C19-H19A } & 109.02(19) \\ \text { N3-C19-H19B } & 109.00(18) \\ \text { N3-C19-C20 } & 111.77(18) \\ \text { H19A-C19-H19B } & 109.0(4) \\ \text { H19A-C19-C20 } & 109.01(18) \\ \text { H19B-C19-C20 } & 109.01(18) \\ \text { C19-C20-H20A } & 109.46(3) \\ \text { C19-C20-H20B } & 109.49(3) \\ \text { C19-C20-H20C } & 109.46(17) \\ \text { H20A-C20-H20B } & 109.47(18) \\ \text { H20A-C20-H20C } & 109.47(2) \\ \text { H20B-C20-H20C } & 109.49(2) \\ \text { H1WA-O1W-H1WB } & 99.3(9) \\ \text { H2WA-O2W-H2WB } & 97.3(8)\end{array}$

Hydrogen-bond geometry $\left(\AA,{ }^{\circ}\right)$

\begin{tabular}{lllll}
\hline$D-\mathrm{H} \cdots A$ & $D-\mathrm{H}$ & $\mathrm{H} \cdots A$ & $D \cdots A$ & $D-\mathrm{H} \cdots A$ \\
\hline $\mathrm{N} 1-\mathrm{H} 1 \mathrm{~N} \cdots \mathrm{Cl} 2^{\mathrm{i}}$ & $0.93(1)$ & $2.26(1)$ & $3.160(6)$ & $162(1)$ \\
$\mathrm{N} 2-\mathrm{H} 2 \mathrm{~N} \cdots \mathrm{O} 2 \mathrm{~W}^{\mathrm{ii}}$ & $0.93(2)$ & $2.07(2)$ & $2.902(12)$ & $148(1)$ \\
$\mathrm{N} 3-\mathrm{H} 3 \mathrm{~N} \cdots \mathrm{Cl} 3$ & $0.93(1)$ & $2.15(1)$ & $3.016(7)$ & $155(1)$ \\
$\mathrm{O} 1-\mathrm{H} 1 \mathrm{O} \cdots \mathrm{Cl} 2^{\mathrm{iii}}$ & 0.98 & 2.06 & $3.037(7)$ & 177 \\
$\mathrm{O} 1 \mathrm{~W}-\mathrm{H} 1 \mathrm{WA} \cdots \mathrm{Cl}$ & 0.97 & 2.16 & $3.127(11)$ & 175 \\
$\mathrm{O} 1 \mathrm{~W}-\mathrm{H} 1 \mathrm{WB} \cdots \mathrm{Cl}{ }^{\mathrm{iv}}$ & 1.02 & 2.28 & $3.297(11)$ & 177 \\
$\mathrm{O} 2 \mathrm{~W}-\mathrm{H} 2 \mathrm{WA} \cdots \mathrm{O} 1 \mathrm{~W}$ & 0.98 & 1.84 & $2.816(14)$ & 171 \\
$\mathrm{O} 2 \mathrm{~W}-\mathrm{H} 2 \mathrm{WB}^{\mathrm{iv}} \mathrm{Cl} 2^{\mathrm{iv}}$ & 1.06 & 2.13 & $3.188(12)$ & 177 \\
$\mathrm{C} 2-\mathrm{H} 2 \cdots \mathrm{O} 2 \mathrm{~W}^{\mathrm{ii}}$ & $0.93(1)$ & $2.37(1)$ & $3.279(12)$ & $167(1)$ \\
$\mathrm{C} 5-\mathrm{H} 5 \cdots \mathrm{Cl} 2^{\mathrm{i}}$ & $0.93(1)$ & $2.77(1)$ & $3.529(9)$ & $140(1)$ \\
$\mathrm{C} 16-\mathrm{H} 16 \mathrm{~B} \cdots \mathrm{O} 1$ & $0.97(1)$ & $2.41(1)$ & $2.813(7)$ & $105(1)$ \\
\hline
\end{tabular}

Symmetry codes: (i) $-x+2,-y+1,-z+1$; (ii) $-x+1,-y+1,-z+2$; (iii) $x,-y+3 / 2, z+1 / 2$; (iv) $-x+1,-y+1,-z+1$. 